\title{
Diffusionsprozesse von Öko-Innovationen
}

\author{
Ein agentenbasierter Ansatz
}

Florian Lewalder, Lehrstuhl für Makroökonomik, Ruhr-Universität Bochum, Universitätsstraße 150, 44801 Bochum

(florian.lewalder@rub.de), (1) orcid.org/0000-0003-4128-6933

Der Artikel diskutiert Hindernisse für einen Strukturwandel zu einer ökologisch nachhaltigen Wirtschaftsweise auf der Produktebene. In einem agentenbasierten Modell wird eine Marktsituation mit Lock-in-Effekten simuliert, welche das technologische Paradigma eines verschmutzenden Produktionsprozesses gegenüber einer neu in den Markt eintretenden Öko-Innovation festigen. Konsumenten haben die Möglichkeit, über ihre Konsumwahl Einfluss auf den Strukturwandel zu nehmen. Dazu unterscheiden sie die ihnen angebotenen Produkte aufgrund von räumlichen, sozialen, ökonomischen und ökologischen Kriterien. Simulationen des Modells zeigen den Einfluss heterogener und homogener Konsumpräferenzen auf das Niveau an Umweltverschmutzung und die Adoptionsraten des umweltfreundlichen Produktes auf.

\section{Diffusion Processes of Eco-Innovations \\ An Agent-Based Approach}

The article discusses product market hindrances for structural change towards an ecologically sustainable economic system. An agent-based model is presented which simulates an economy affected by path dependencies and lock-in effects. A new environmentally friendly product enters this market and tries to break the technological paradigm of a pollutive production technology. Consumers can influence this process of structural change via their consumption decision which depends on an assessment of products by means of spatial, social, economic, and ecological criteria. Simulations show how the model results, namely the speed of adoption and the level of pollution over time, are influenced by heterogeneous and homogeneous consumption preferences.

KEYWORDS: eco-innovation, diffusion, technological paradigm, structural change, heterogeneity

This is an article distributed under the terms of the Creative Commons Attribution License CCBY 4.0 (https://creativecommons.org/licenses/by/4.0/)

https://doi.org/10.14512/tatup.26.3.30

Submitted: 05. 07.2017. Peer reviewed. Accepted: 04.10.2017

\section{Einleitung}

Ökologische Herausforderungen durch Schadstoffe, die als Nebenprodukte industrieller Fertigung anfallen, werden vermehrt als gesellschaftliches Problem anerkannt. Dies gilt nicht nur für den Kohlenstoffdioxidausstoß und den dadurch beschleunigten Klimawandel, sondern auch für Verunreinigungen, die durch Schwermetalle, Pestizide, Feinstaub und viele weitere Schadstoffe verursacht werden. Der Verbraucher hat durch die Wahl seines persönlichen Konsums prinzipiell die Möglichkeit, erheblichen Einfluss auf Umweltzerstörung und -verschmutzung auszuüben. Für viele Produkte des alltäglichen Konsums existieren grüne Alternativen, jedoch bleibt deren Marktdurchdringung häufig hinter den Erwartungen zurück. Studien weisen darauf hin, dass Konsumenten in Entscheidungssituationen auch dann nicht zu umweltfreundlichen Produkten greifen, wenn sie selbst eine positive Einstellung gegenüber Umweltthemen haben (Ozaki 2011). Wenn der Strukturwandel hin zu einem ökologisch-nachhaltigen technologischen Paradigma gelingen soll, ist es unabdingbar zu verstehen, wie Konsumenten durch ihre individuelle Konsumnachfrage dazu beitragen können und wo potenzielle Hindernisse für die Marktdurchdringung eines solchen von der Nachfrageseite induzierten Strukturwandels entstehen können. Agentenbasierte Modelle können hier einen großen Beitrag leisten. Sie bieten die Möglichkeit, ökonomische, soziologische und psychologische Intuition direkt zu modellieren und auf dieser Grundlage das Individualverhalten einzelner Haushalte zu simulieren. Durch Aggregation der Ergebnisse der Individualebene lassen sich wiederum Schlüsse für makroökonomische und gesamtgesellschaftliche Phänomene und Szenarien ziehen.

In diesem Beitrag wird ein agentenbasiertes Modell genutzt, um den Einfluss heterogener Konsumpräferenzen auf Diffusionsprozesse umweltfreundlicher Produkte zu analysieren. Dazu wird zunächst kurz auf Eigenschaften von Öko-Innovationen eingegangen, die diese von anderen Innovationen unterscheiden und den Markterfolg erschweren können sowie ein kurzer Überblick über die agentenbasierte Diffusionsliteratur gegeben. Danach wird das Modell vorgestellt, welches sich mit der Einführung von 
Öko-Innovationen in Marktsituationen befasst, die durch Lockin-Effekte geprägt sind. Anhand von Simulationsergebnissen des Modells werden die Einflüsse heterogener und homogener Konsumpräferenzen verglichen. Das kurze Fazit resümiert die Ergebnisse und empfiehlt zwei mögliche Förderinstrumente für Öko-Innovationen.

\section{Diffusionsprozesse von Öko-Innovationen und ABM}

Umweltfreundliche Technologien, Produktionsprozesse und Endprodukte weisen spezifische Eigenschaften auf, die bei der erfolgreichen Etablierung am Markt hinderlich sind (Cecere et al. 2014). Sie unterscheiden sich dabei teilweise von anderen Innovationen, bei denen der Aspekt der Umweltfreundlichkeit nicht im Vordergrund steht. Für die Entstehung von Öko-Innovationen spielen positive externe Effekte, also positive Nebenwirkungen ökonomischer Entscheidungen auf andere Marktteilnehmer sowohl in der Entwicklungs- als auch in der Diffusionsphase eine wichtige Rolle (Rennings 2000). In der Entwicklungsphase kann das bedeuten, dass Marktkonkurrenten durch Wissenstransfer davon profitieren, wenn ein Unternehmen in die Entwicklung öko-innovativer Technologien investiert. Das reduziert die Anreize von Unternehmen, in die Erforschung dieser Technologien zu investieren. Die externen Effekte in der Diffusionsphase beziehen sich auf positive Umweltexternalitäten, wie beispielsweise umweltschädliche Emissionen im Produktionsprozess. Die Gesellschaft profitiert von Öko-Innovationen durch die Verbesserung der Umwelt, während das Unternehmen die Kosten übernehmen muss, die mit der Einhaltung von Umweltrichtlinien und der Reduzierung von anfallender Verschmutzung einhergehen. Dies verringert zusätzlich die Anreize zur Investition in umweltfreundliche Technologien, da Unternehmen, die eine solche Investition tätigen wollen, höhere Kosten in Kauf nehmen müssen als ihre direkte Konkurrenz, die weiterhin in konventionelle Technologie investiert.

Auch bei der individuellen Konsumentscheidung spielen die Umweltexternalitäten eine Rolle. So mag die Wahl eines umweltfreundlichen Produktes zwar gesellschaftlich von hohem Nutzen sein, für den privaten Konsumenten jedoch kann in vielen Fällen weiterhin das konventionelle Produkt die bessere Alternative sein. Unternehmen haben daher Schwierigkeiten, den sozialen Nutzen, der sich gesamtgesellschaftlich in Form von Umweltfreundlichkeit einer Innovation auswirkt, am Markt vom einzelnen Konsumenten auch monetär vergütet zu bekommen. Auf dieser Grundlage werden Investitionen in Öko-Innovationen eher widerwillig getätigt. Unternehmen und Konsumenten, die sich für umweltfreundliche Produkte entscheiden, müssen Kompromisse eingehen zwischen der Ökobilanz und Preis-/ Qualitätsfaktoren (Oltra und Saint Jean 2009). Solange umweltschädliche Auswirkungen konventioneller Produktionstechnologien am Markt weder durch den Konsumenten bestraft noch durch die Politik reguliert werden, ist der Wettbewerb zwischen umweltfreundlichen und umweltschädlichen Technologien verzerrt.

Ein weiteres Problem für die Entwicklung von Öko-Innovationen können technologische Paradigmen darstellen, die aufgrund von Lernkurven, Skalenerträgen, existierender Infrastruktur oder der Gewöhnung an einen bestimmten Lebensstil einer Gesellschaft zu Pfadabhängigkeiten und Lock-in-Effekten führen (Rennings 2000). Letztlich hängt die Entwicklung umweltfreundlicher Technologien auch von einer Sensibilität für das Thema Umwelt ab, sowohl auf Seite der Produzenten als auch auf Seite der Konsumenten. Hier spielt die eigene Erfahrung mit Umweltverschmutzung potenziell eine wichtige Rolle. So deuten Ergebnisse aus Fallstudien darauf hin, dass Konsumenten, die selbst durch Umweltverschmutzung beeinträchtigt sind, eher zum Kauf umweltfreundlicher Produkte neigen als Konsumenten, die selbst weder Umweltverschmutzung noch deren Folgen ausgesetzt sind (Windrum et al. 2009).

Zur Analyse von Öko-Innovationen bietet sich ein interdisziplinärer Ansatz an, da sich die Materie zwischen der Innovationsund Umweltökonomik sowie den Sozialwissenschaften verorten lässt. In einem umfangreichen Übersichtsartikel stellen Kiesling et al. (2012) fest, dass die klassisch aggregierten Diffusionsmodelle nur eine eingeschränkte Zahl eher theoretischer Fragestellungen beantworten können und wegen geringer Erklärungskraft in der Kritik stehen. Ein Beispiel ist der auf Rogers (2003) zurückgehende Ansatz, fünf unterschiedliche Gruppen von Individuen anhand ihrer Adoptionsneigung bei Innovationen zu definieren: Innovatoren, Early Adopter, frühe sowie späte Mehrheit und Nachzügler. Diese Gruppen werden jedoch nicht explizit modelliert. Stattdessen wird das aggregierte Adoptionsverhalten bei einer Innovation in Form einer S-Kurve dargestellt, um diese anschließend in unterschiedliche Abschnitte zu unterteilen, die dann als oben genannte Konsumentengruppen beschrieben werden. Mit dieser Form lassen sich Diffusionsprozesse zwar beschreiben, es fehlt jedoch die Möglichkeit zu analysieren, weshalb sich einzelne Individuen für die Adoption der Innovation entscheiden und wie sie sich untereinander dabei beeinflussen. So heben Kiesling et al. (2012) dann auch hervor, dass Heterogenität unter Konsumenten sowie komplexe Dynamiken sozialer Prozesse einen starken Einfluss auf Diffusionsprozesse haben. Gleichzeitig beobachten sie, dass die Methode der agentenbasierten Modellierung in der Diffusionsliteratur seit einigen Jahren immer stärker genutzt wird. Durch den dortigen Perspektivwechsel - weg vom sozialen System als Ganzes und hin zum einzelnen Konsumenten und dessen individuellen Eigenschaften, sozialen Interaktionen und Entscheidungsprozessen - können sich in agentenbasierten Modellen Dynamiken auf der Makroebene herausbilden, die ihren Ursprung im aggregierten Individualverhalten und in der komplexen Interaktion zwischen Individuen haben (Kiesling et al. 2012).

Im folgenden Abschnitt wird ein räumliches agentenbasiertes Modell näher beschrieben, welches die Probleme von Öko-Innovationen beim Eintritt in einen Markt untersucht, der von Lock-in-Effekten geprägt ist. 


\section{Modell}

Das Modell untersucht Auswirkungen heterogener Konsumpräferenzen auf die Nachfrage und die Diffusion von Öko-Innovationen. Es umfasst zwei Agentenklassen - die unten vorgestellten Unternehmen und Haushalte -, welche zufällig im zweidimensionalen Raum verteilt werden, und betrachtet eine Marktsituation, in der alteingesessene Unternehmen eine traditionelle, verschmutzende Technologie zur Produktion eines Konsumguts nutzen.

\section{Unternehmen}

Eine erste Gruppe von Unternehmen ist am Markt etabliert. Diese haben die räumlichen Nischen weitgehend gefüllt und profitieren aufgrund von Lernkurven von einer steigenden Produktivität und einer wachsenden Qualität ihrer Produkte. Eine neue Technologie erlaubt es nun einer zweiten Gruppe neugegründeter Unternehmen, ihre Produkte ohne anfallende Umweltverschmutzung zu produzieren. Um sich am Markt erfolgreich aufstellen zu können, müssen diese öko-innovativen Unternehmen das technologische Paradigma brechen. Die Produktionsfunktionen der beiden Technologien sind identisch und berücksichtigen die Produktivität des Unternehmens sowie die Arbeitskraft von Arbeiternehmern als einzigen Produktionsfaktor. Unternehmen können nicht zwischen den Produktionstechnologien wechseln und sind heterogen bezüglich ihrer Arbeitsproduktivität und der Qualität ihrer Produkte. Die Lernkurven dieser beiden Faktoren sind hingegen unterschiedlich. Während die Produktivität eines einzelnen Unternehmens von der Menge aller jemals von ihm produzierten Produkte abhängt, wird die Produktqualität von der Zeitspanne bestimmt, die das Unternehmen bereits am Markt tätig ist.

Neu in den Markt eintretende Unternehmen haben durch hohe Produktionszahlen die Möglichkeit, langfristig eine höhere Produktivität als alteingesessene Unternehmen zu erreichen. Dies gilt nicht für die Produktqualität, da diese nicht von der Produktionsmenge abhängt. Es wird davon ausgegangen, dass Arbeitsprozesse umso schneller und effizienter ausgeführt werden, je häufiger sie schon ausgeführt wurden. Die Modellierung der qualitätsbezogenen Lernkurve spiegelt den in der Literatur diskutierten Umstand wider, dass der soziale Nutzen von Öko-Innovationen größer ist als der private Nutzen (Rennings 2000).

Um einen Lock-in-Effekt auf dem Markt zu erzeugen, existieren zu Beginn der Simulation nur konventionelle Produzenten, während öko-innovative Unternehmen erst im Zeitverlauf gegründet werden. Die beiden Lernkurven führen so zu den bereits erwähnten Wettbewerbsvorteilen für die schon länger am Markt etablierten konventionellen Unternehmen. Weiter verstärkt wird der Lock-in-Effekt durch die Tendenz der Haushalte, sich bei der Konsumentscheidung am sozialen Umfeld zu orientieren. Letzteres stellt für neue Unternehmen eine weitere Hürde dar, da sie sich somit auch gegen den sozialen Druck zur Konformität behaupten müssen.

\section{Haushalte}

Die Haushalte bieten ihre Arbeitskraft auf dem Arbeitsmarkt an und nutzen ihr Einkommen für Konsumzwecke. Sie haben eine eingeschränkte Reichweite und können nur bei Unternehmen in einem Radius um den eigenen Standort einkaufen oder arbeiten. ${ }^{1}$ Durch ihre Konsumwahl haben sie die Möglichkeit, der neuen, umweltfreundlichen Technologie am Markt zum Durchbruch zu verhelfen. Dazu wird ihnen eine einfache Regel zum Vergleich von Produkten an die Hand gegeben. Die Entscheidung für ein Konsumgut basiert auf einer gewichteten Summe, welche folgende fünf Kriterien mit einfließen lässt: Distanz zwischen Haushalt und Unternehmen, Konsumentscheidungen im nahen sozialen Umfeld, Preis, Produktqualität und Umweltschädlichkeit des Produktionsprozesses.

Haushalte evaluieren alle Produkte, die für sie verfügbar sind, anhand dieser Funktion, ordnen ihnen einen subjektiven Wert zu und sortieren diese dann hierarchisch, beginnend mit dem am besten bewerteten Konsumgut. Das Distanzkriterium führt zu räumlichem Wettbewerb, vergleichbar zu klassischen Modellen der Volkswirtschaftslehre (Hotelling 1929). Bei der sozialen Dimension achten die Haushalte auf den Konsum in ihrer direkten Nachbarschaft. Je mehr Nachbarn konventionelle (bzw. öko-innovative) Produkte konsumieren, umso mehr wollen Haushalte ebenfalls ein konventionelles (bzw. öko-innovatives) Produkt konsumieren. Bei der Bewertung von Preis und Qualität eines Konsumguts werden die Preise und Produktqualitäten aller Unternehmen in der Reichweite eines Haushalts verglichen. Je niedriger der Preis und je höher die Qualität eines Produktes, umso besser wird es bewertet. Weiter geht die Umweltschädlichkeit des Produktionsprozesses mit in die Entscheidungsregel ein. Dazu nutzen die Haushalte eine logistische Funktion, um das aktuelle Ausmaß an globaler Verschmutzung zu evaluieren, welche sich über die Zeit mit jedem umweltschädlich produzierten Gut akkumuliert und von der Umwelt nur langsam absorbiert werden kann. Diese funktionale Form entspricht stilisierten Fakten zur Adoption von Öko-Innovationen (Windrum et al. 2009) und spiegelt wider, dass Individuen sich in Entscheidungssituationen häufig nur für umweltfreundliche Produkte entscheiden, wenn sie selbst von Verschmutzung oder deren Folgen betroffen sind.

Zur Untersuchung des Einflusses von Heterogenität der Konsumpräferenzen auf die Modellergebnisse dienen die Gewichtungen der unterschiedlichen Kriterien in der Entscheidungsfunktion. Bei heterogenen Präferenzen werden vier unterschiedliche Agententypen genutzt: soziale, preissensitive, qualitätsmaximierende und umweltorientierte Konsumenten. Diese legen auf jeweils ein Kriterium besonders viel Wert (Gewichtung von $60 \%$ ), während die anderen Kriterien nur eine untergeordnete Rolle spielen (Gewichtung von jeweils 10\%). In der Simulation mit heterogenen Konsumpräferenzen werden jedem Haushalt die Präferenzen eines dieser vier Agententypen mit jeweils gleicher Wahrscheinlichkeit zugewiesen. Im Fall von

1 Hier wird angenommen, dass die Transportkosten eines Produktes oder die Fahrtkosten eines Haushaltes ab einer gewissen Distanz prohibitiv hoch sind 
homogenen Präferenzen werden dahingegen alle Kriterien gleich gewichtet.

\section{Ablaufplan}

Um ein besseres Bild davon zu bekommen, was genau in welcher Reihenfolge simuliert wird, folgt nun eine kurze Übersicht über den Ablaufplan des Modells:

1. Initialisierung: Haushalte und Unternehmen werden kreiert, Arbeitsbeziehungen zwischen ihnen hergestellt und auf deren Basis die Parameter der Unternehmen berechnet (vergangene Produktion, Lohnniveau, etc.).

2. Qualität und Produktivität der Unternehmen werden aktualisiert.

3. Unternehmen bestimmen ihre Arbeitsnachfrage, entlassen gegebenenfalls

Arbeitskräfte oder schreiben neue Stellen aus. Haushalte bestimmen ihr Arbeitsangebot. Im Anschluss findet ein Matching-Prozess auf dem Arbeitsmarkt statt.

4. Unternehmen bestimmen anhand ihres aktuellen Lohnniveaus, ihrer Produktivität pro eingesetzter Arbeitskraft und ihres individuellen Preisaufschlags ihren Preis.

5. Unternehmen produzieren Güter. Anfallende Verschmutzung wird an die Umwelt abgegeben.

6. Haushalte gehen nacheinander einkaufen. Sie bewerten alle verfügbaren Produkte anhand ihrer Entscheidungsregel und kaufen das bestbewertete Produkt.

7. Löhne werden ausgezahlt. Unternehmen aktualisieren ihre Ersparnisse und verlassen im Insolvenzfall den Markt. Gewinnüberschüsse werden in Form von Dividenden gleichmäBig unter den Haushalten aufgeteilt.

8. Neue Unternehmen treten dem Markt bei. Sie führen von einem zufälligen Punkt im zweidimensionalen Raum aus eine Marktanalyse durch. Wenn das Unternehmen erwartet, eine ausreichende Menge seiner Produkte absetzen zu können, gründet sich an dieser Stelle ein neues Unternehmen.

9. Wiederholung der Punkte 2-8 über 1000 Simulationsrunden.

\section{Simulationsergebnisse}

Die folgenden Modellergebnisse basieren auf Durchschnittswerten von jeweils 80 Simulationen für die beiden Szenarien mit homogenen bzw. heterogenen Konsumpräferenzen der Haushalte. Es soll untersucht werden, welchen Einfluss die Heterogenität von Konsumpräferenzen auf die Verbreitung des umweltfreundlichen Konsumguts hat. Im Fall des vorliegenden agentenbasierten Modells kann durch die direkte Modellierung von Agentenverhalten genau untersucht werden, wie Haushalte ihre Entscheidungen treffen, sich dabei gegenseitig beeinflussen und so die aggregierte Adoptionskurve bilden.
Das Diagramm in Abbildung 1 zeigt, wie sich die Adoptionskurve der Öko-Innovation in den Fällen homogener und heterogener Konsumpräferenzen über die Zeit verändert. Die beiden fett markierten Graphen im Diagramm zeigen die gesamtgesellschaftlichen Adoptionsraten für den Heterogenitäts- und Homogenitätsfall, während die schmalen Linien die Adoptionsraten der vier Gruppen von Konsumtypen bei Heterogenität darstellen. Es fällt auf, dass sich die Dynamiken teilweise deutlich voneinander unterscheiden. Obwohl die gesamtgesellschaftliche Adoptionsrate bei heterogenen Präferenzen anfangs schneller steigt als im Homogenitätsfall, entwickelt sie sich bei Homogenität mittel- und langfristig besser. Im Folgenden werden zunächst der Heterogenitätsfall und anschließend der Homogenitätsfall näher beleuchtet.

\section{Heterogene Präferenzen}

Der anfängliche Anstieg konsumierter Öko-Innovationen bei Heterogenität wird fast ausschließlich von umweltorientierten Konsumenten getragen, während soziale, preissensitive und qualitätsmaximierende Konsumenten deutlich länger bei konventionell produzierenden Unternehmen einkaufen (siehe Abbildung 1). Dies hat unterschiedliche Gründe: Einerseits können Veränderungen im Konsumverhalten auf Gründungsaktivitäten umweltfreundlicher Unternehmen und leere Lagerbestände konventioneller Unternehmen zurückgehen. Im oberen rechten Diagramm von Abbildung 2 ist zu erkennen, dass insbesondere im Heterogenitätsfall schon früh und in starkem Maße umweltfreundliche Unternehmen gegründet werden (graue Linie). Gestützt werden diese durch die Nachfrage der umweltorientierten Konsumenten. Andererseits ändern die unterschiedlichen Konsumtypen ihre Nachfrage, wenn bestimmte Voraussetzungen geschaffen wurden. Zwar spielen weiter alle fünf Entscheidungskriterien für alle Haushalte eine Rolle bei der Konsumentschei- 

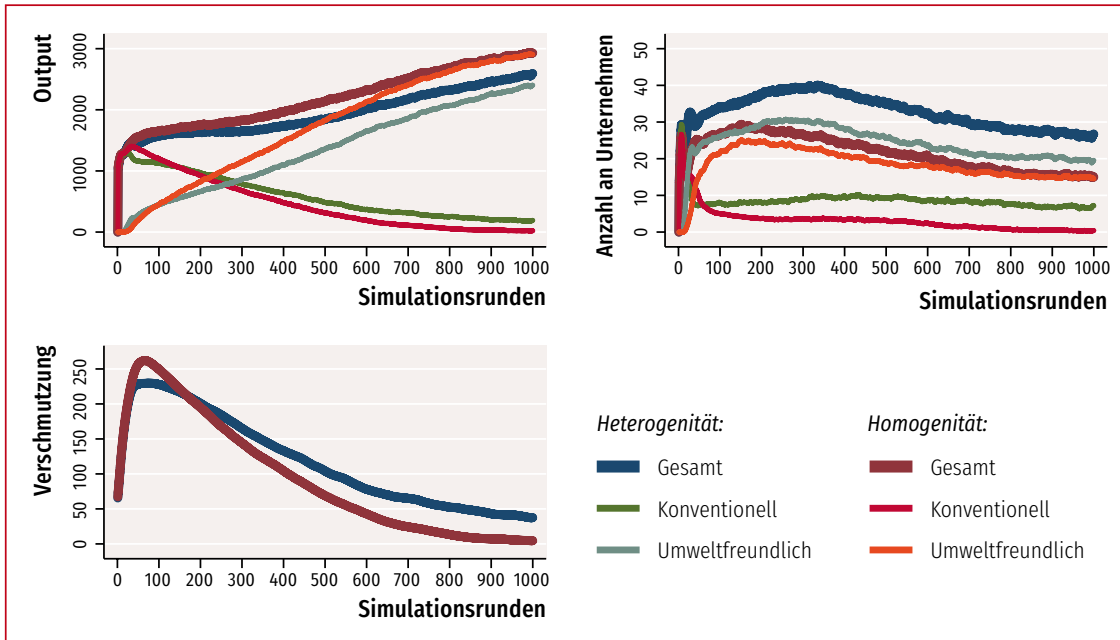

Abb.2: Produzierter Output, Anzahl der Unternehmen und Umweltverschmutzung im Zeitverlauf.

Quelle: Eigene Darstellung

dung, aber die starke Neigung zu jeweils einem Kriterium führt zu großen Unterschieden im Konsumverhalten über die Zeit. Abbildung 3 zeigt hierzu, wie die unterschiedlichen Konsumentengruppen die von ihnen konsumierten Produkte subjektiv bewerten. Die großen Unterschiede in der Bewertung der unterschiedlichen Kriterien gehen auf die mit einbezogenen Gewichtungen zurück. So legen umweltorientierte Haushalte sehr viel Wert auf das Umweltkriterium, weshalb die subjektive Bewertung eines Produktes auch maßgeblich von diesem Kriterium beeinflusst wird. Jedoch sinkt im Laufe der Simulation die Umweltverschmutzung wieder (siehe Abbildung 2 unten links), da immer weniger umweltschädliche Produkte produziert werden und die Umwelt einen Teil der Verschmutzung absorbieren kann. Infolgedessen sinkt langfristig die Relevanz des Umweltkriteriums bei der Konsumentscheidung für alle Konsumenten. Dies erklärt auch, warum ursprünglich umweltorientierte Konsumenten langfristig nicht zwangsläufig beim umweltfreundlichen Produkt bleiben, wie in Abbildung 1 zu sehen ist (grüne Linie).

\section{Langfristig sinkt die Relevanz des Umweltkriteriums bei der Konsumentscheidung.}

\section{Soziale Konsumenten}

Soziale Konsumenten tendieren dazu, ihre Konsumwahl zu ändern, wenn eine kritische Masse von Agenten in ihrem sozialen Umfeld bereits zur Öko-Innovation gewechselt ist. Die Kombination aus umweltorientierten Nachbarn und, wie im unteren linken Diagramm von Abbildung 2 (blaue Linie) zu sehen, einer stark ansteigenden Verschmutzung zu Beginn der Simulation, moti- viert eine steigende Zahl sozialer Konsumenten, zu Öko-Innovationen zu wechseln. In Abbildung 3 ist erkennbar, dass die zwischenzeitlich gesunkene, subjektive Bewertung des sozialen Kriteriums der Produkte, die von sozialen Haushalten konsumiert werden, wieder ansteigt, wenn etwa ab Periode 220 die Adoptionsrate in der Gesamtbevölkerung $50 \%$ überschreitet. Das bedeutet, dass soziale Konsumenten beginnen, die Öko-Innovation als neuen Standard zu betrachten.

\section{Preissensitive Konsumenten}

Ein anderes Bild ergibt sich für die preissensitiven Haushalte. Zwar spüren auch diese Konsumenten anfangs die steigende Umweltverschmutzung, jedoch fokussieren sie sich mit dem Preis auf ein Entscheidungskriterium, das neu eintretende umweltfreundliche Unternehmen aufgrund der Lernkurve für Arbeitsproduktivität nur langfristig für sich entscheiden können. Wie das obere linke Diagramm in Abbildung 2 zeigt, übersteigt der produzierte Output umweltfreundlicher Unternehmen (graue Linie) nach einer gewissen Zeit den Output konventionell produzierender Unternehmen (grüne Linie) deutlich, was mittel- und langfristig steigende Produktivität impliziert. ${ }^{2}$ Da die Lernkurven nur für einzelne Unternehmen und nicht für den gesamten Sektor gelten, stellen die von der Produktivität abhängenden, vergleichsweise hohen Preise für umweltfreundliche Produkte für einen langen Zeitraum ein Hindernis für preissensitive Konsumenten dar.

\section{Qualitätsmaximierende Konsumenten}

Die Konsumtypen, die am längsten beim Konsum konventioneller Produkte bleiben, sind die qualitätsmaximierenden Haushalte. Sie passen ihren Konsum, wie in Abbildung 1 (schwarze Linie) deutlich zu erkennen, nur widerwillig an, da die konventionellen Unternehmen auch langfristig ein qualitativ hochwertigeres Produkt anbieten. Der Grund hierfür ist die, lediglich von der Zeit abhängige, Lernkurve für Produktqualität. Sie verschafft konventionellen Firmen auch dann noch einen leichten Vorteil in Bezug auf Qualität, wenn die umweltfreundlichen Unternehmen bereits einen Großteil des Markts dominieren. Die beinahe horizontal verlaufende Kurve für die Bewertung des Qualitätskriteriums in Abbildung 3 (unten rechts), weist darauf hin, dass die qualitätsmaximierenden Haushalte über weite Teile der Simulation ausschließlich zu den Produkten mit der jeweils höchsten Qualität greifen. Dass diese Haushalte letztlich dennoch vermehrt zum umweltfreundlichen Produkt greifen, hat sowohl mit sozialen Effekten und potenziell geringeren Preisen der

2 Einschränkend muss allerdings hinzugefügt werden, dass - abgesehen vom Beginn der Simulation - mehr als doppelt so viele umweltfreundliche Unternehmen im Spiel sind als konventionelle. 
umweltfreundlichen Produkte zu tun als auch mit zunehmenden Marktaustritten von nicht mehr wettbewerbsfähigen, konventionellen Unternehmen und der daraus resultierenden potenziellen Knappheit an hochqualitativen, konventionellen Produkten.

\section{Homogene Präferenzen}

Für den Fall homogener Konsumpräferenzen geht aus Abbildung 2 zwar ein höheres Maximalniveau an Umweltverschmutzung hervor als im Heterogenitätsfall, jedoch sinkt dieses stärker im Zeitverlauf und auch die Adoptionsrate ist nach Ablauf der Simulation deutlich höher. In Abbildung 4 lassen sich dazu die entsprechenden Dynamiken der subjektiven Bewertungen für die unterschiedlichen Entscheidungskriterien ablesen. Da die Gewichtungen der unterschiedlichen Kriterien im Fall von heterogenen Präferenzen untereinander identisch sind (jeweils $20 \%$ ), sieht man hier eine Überlagerung der Kurven. Zu Beginn der Simulation gewinnt die Umweltverschmutzung dramatisch an Relevanz für das Entscheidungsverhalten der Haushalte. Dies treibt auch die zunächst sprunghaft steigende Gründung von umweltfreundlichen Unternehmen an (siehe Abbildung 2, rechts oben), wobei diese unter den Bedingungen homogen verteilter Konsumpräferenzen langsamer verläuft als unter heterogenen Bedingungen. Anhand der durchweg hohen Bewertung des sozialen Kriteriums lässt sich folgern, dass sich Cluster von Konsumenten konventioneller und umweltfreundlicher Produkte bilden, da die Haushalte ihren gekauften Produkten nur dann eine hohe Bewertung für das soziale Kriterium geben, wenn ein hoher Anteil ihrer Nachbarn ein Produkt mit derselben Eigenschaft (konventionell oder umweltfreundlich) konsumieren. Die sinkende Relevanz der Umweltverschmutzung über die Zeit wird aufgefangen durch die Tatsache, dass nach 200 Zeiteinheiten bereits etwa $60 \%$ der Haushalte zum umweltfreundlichen Konsum gewechselt sind. So wirkt das Umweltkriterium hier ähnlich wie im heterogenen Fall als Treiber des beginnenden Strukturwandels, hat jedoch für alle Haushalte eine relativ hohe Relevanz und nicht nur für eine kleine Gruppe. Die leichte Substituierbarkeit der Entscheidungskriterien unterstützt langfristig den Übergang zur umweltfreundlichen Technologie.
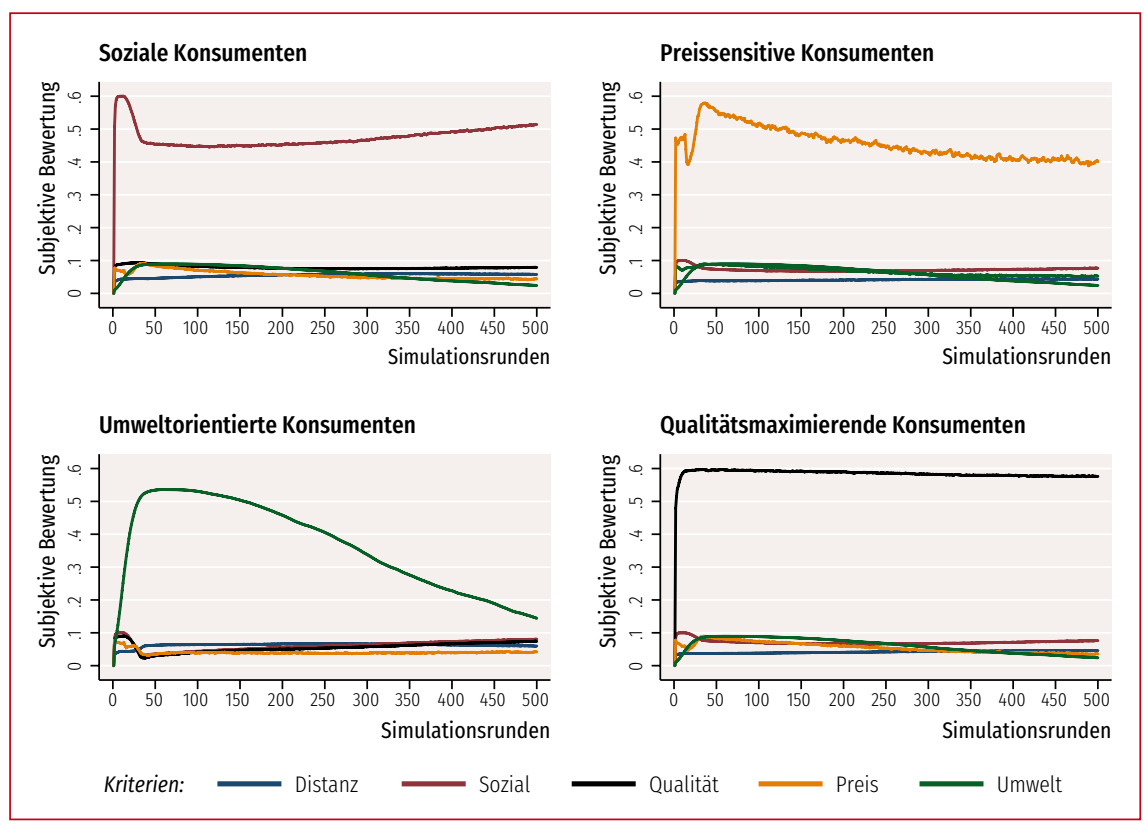

Abb.3: Kriterienbewertung im Zeitverlauf, unterteilt in Konsumtypen.

Quelle: Eigene Darstellung

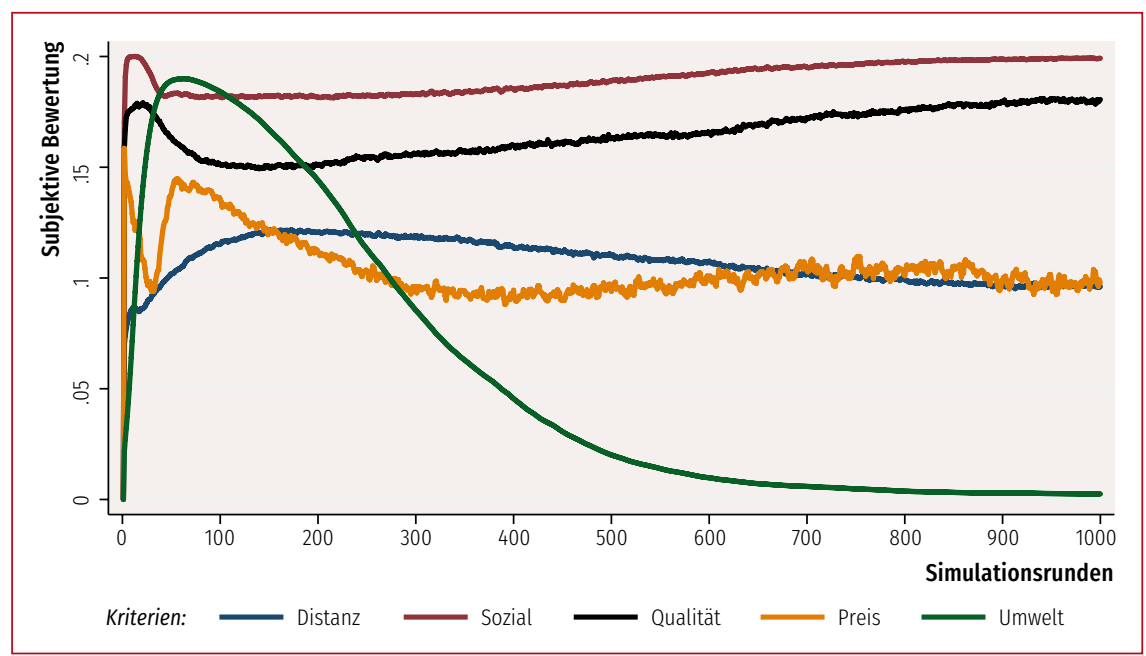

Abb.4: Subjektive Kriterienbewertung bei Homogenität der Konsumpräferenzen. Quelle: Eigene Darstellung

\section{Fazit}

In der vorgestellten Analyse wurde ein agentenbasiertes Modell genutzt, um den Einfluss heterogener und homogener Konsumpräferenzen auf den Diffusionsprozess einer umweltfreundlichen Innovation zu untersuchen. Die Ergebnisse zeigen, dass sich die Adoptionsdynamiken sowohl auf Individualebene wie auch auf gesellschaftlicher Ebene deutlich verändern, je nachdem ob heterogene oder homogene Konsumpräferenzen der Modellierung zugrunde gelegt. Unter heterogenen Bedingungen führten die verschiedenen Schwerpunkte bei der Konsumentscheidung einerseits zu einer schnelleren Diffusion der öko-innovativen Pro- 
dukte in der Frühphase der Simulation, andererseits jedoch zu einer verringerten langfristigen Diffusion. Diese Unterschiede ließen sich insbesondere an zwei der vier Konsumentengruppen festmachen: den umweltorientierten und qualitätsmaximierenden Haushalten. Das Ergebnis zeigt auf, wie wichtig die genaue $\mathrm{Zu}-$ sammensetzung einer Gesellschaft für die Diffusionsdynamik von Öko-Innovationen ist und schärft das Verständnis für nachfrageseitige Beschleuniger sowie Hindernisse eines Strukturwandels zu einer nachhaltigeren Wirtschaftsweise.

Die Unterteilung der Gesellschaft, hier beispielhaft in vier Gruppen, ergibt, verglichen mit einer aggregierten Auffassung von Konsumverhalten, ein differenzierteres Bild davon, wie einzelne Konsumgruppen adressiert werden können. Die Modellergebnisse implizieren zwei Empfehlungen für Politikmaßnahmen mit dem Ziel einer schnelleren Diffusion von Öko-Innovationen. Eine Subvention für Öko-Innovationen könnte sich senkend auf den Preis auswirken und so eine schnellere Adoption des neuen Produktes seitens der preissensitiven Haushalte nach sich ziehen. Über soziale Effekte hätte dies Auswirkungen auf die gesamte Gesellschaft. Alternativ könnte sich ein Förderinstrument in Form einer staatlichen Nachfrage nach dem öko-innovativen Produkt sowohl vorteilhaft auf die Lernkurven der öko-innovativen Unternehmen auswirken als auch das Risiko senken, dass ein junges umweltfreundliches Unternehmen durch den starken Wettbewerb aus dem Markt gedrängt wird. Beide Politikmaßnahmen können anhand des hier vorgestellten Modells simuliert und ihr Einfluss auf das Konsumverhalten sowie die Marktdurchdringung der Öko-Innovation analysiert werden.

\section{Literatur}

Cecere, Grazia; Corrocher, Nicoletta; Gossart, Cédric; Ozman, Muge (2014):

Lock-in and Path Dependence: An Evolutionary Approach to Eco-innovations.

In: Journal of Evolutionary Economics 24 (5), S. 1037-1065. DOI: 10.1007/ s00191-014-0381-5.
Hotelling, Harold (1929): Stability in Competition. In: The Economic Journal 39

(153), S. 41-57. DOI: 10.2307/2224214.

Kiesling, Elmar; Günther, Markus; Stummer, Christian; Wakolbinger, Lea (2012): Agent-Based Simulation of Innovation Diffusion: A Review. In: Central European Journal of Operations Research 20 (2), S. 183-230. DOI: 10.1007/ s10100-011-0210-y.

Oltra, Vanessa; Saint Jean, Maïder (2009): Sectoral Systems of Environmental Innovation: An Application to the French Automotive Industry. In: Technological Forecasting and Social Change 76 (4), S. 567-583. DOI: 10.1016/j. techfore.2008.03.025.

Ozaki, Ritsuko (2011): Adopting Sustainable Innovation: What Makes Consumers Sign Up to Green Electricity? In: Business Strategy and the Environment 20 (1), S. 1-17. DOI: 10.1002/bse.650.

Rennings, Klaus (2000): Redefining Innovation: Eco-Innovation Research and the Contribution from Ecological Economics. In: Ecological Economics 32 (2), S. 319-332. DOI: 10.1016/S0921-8009(99)00112-3.

Rogers, Everett M. (2003). Diffusion of Innovations. New York: Free Press. Windrum, Paul; Ciarli, Tommaso; Birchenhall, Chris (2009): Consumer Heterogeneity and the Development of Environmentally Friendly Technologies. In: Technological Forecasting and Social Change 76 (4), S. 533-551. DOI: 10.1016/j.techfore.2008.04.011

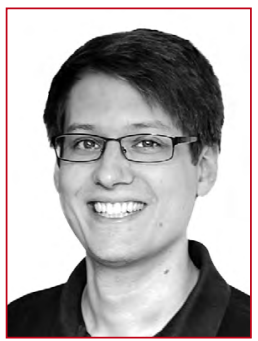

\section{FLORIAN LEWALDER}

ist wissenschaftlicher Mitarbeiter am Lehrstuhl für Makroökonomik der Ruhr-Universität Bochum und forscht zu den Themen Öko-Innovationen, Diffusionsprozesse und agentenbasierte Modellierung.

\section{Sind Sie auf der Suche nach dem passenden Weihnachtsgeschenk?}

Dann haben wir das Richtige für alle Querdenker und Vordenkerinnen:

Mit einem Abo der politischen ökologie verschenken Sie ein Jahr neue Perspektiven und unorthodoxe Lösungsvorschläge für die ökologischen Herausforderungen unserer Zeit!

\section{Das pö-Geschenkabo-Angebot*}

Vier Ausgaben für nur 48,53 Euro statt regulär 64,70 Euro (zzgl. Versandkosten).
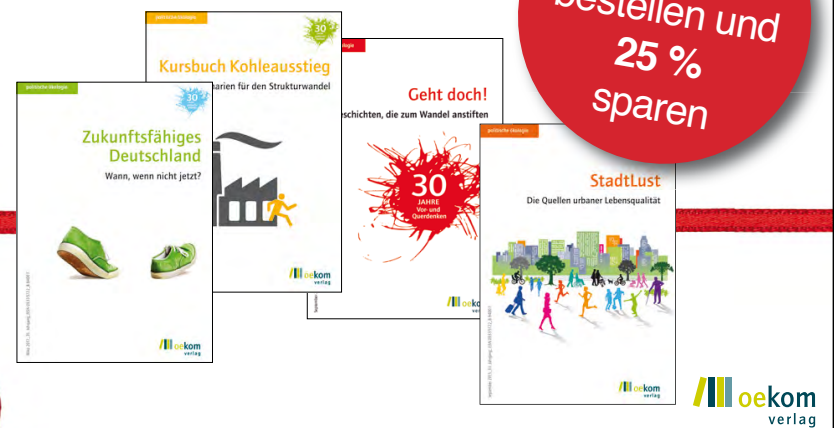